\title{
Type 2 diabetes, socioeconomic status and risk of cancer in Scotland 2001-2007
}

\author{
J. J. Walker • D. H. Brewster • H. M. Colhoun • C. M. Fischbacher • G. P. Leese • \\ R. S. Lindsay • J. A. McKnight • S. Philip • N. Sattar • D. L. Stockton • S. H. Wild • \\ on behalf of the Scottish Diabetes Research Network (SDRN) Epidemiology Group
}

Received: 25 October 2012 /Accepted: 29 April 2013 /Published online: 10 May 2013

(C) Springer-Verlag Berlin Heidelberg 2013

\begin{abstract}
Aims/hypothesis The objective of this study was to use Scottish national data to assess the influence of type 2 diabetes on the risk of cancer at 16 different sites, while specifically investigating the role of confounding by socioeconomic status in the diabetes-cancer relationship.
\end{abstract}

Members of the SDRN Epidemiology Group beyond the authors listed: I. Brady, University of Dundee; J. Chalmers, Victoria Hospital, Kirkcaldy; S. Cunningham, University of Dundee; A. Emslie-Smith, Arthurstone Medical Centre, Dundee; L. Govan, University of Glasgow; B. Guthrie, University of Dundee; D. Levin, University of Dundee; S. Livingstone, University of Dundee; H. Looker, University of Dundee; R. McAlpine, Diabetes Managed Clinical Network, NHS Tayside; A. Morris, University of Dundee; D. Pearson, Aberdeen Royal Infirmary, Aberdeen; J. Petrie, University of Glasgow

J. J. Walker $(\bowtie) \cdot$ S. H. Wild

Centre for Population Health Sciences, The University

of Edinburgh, Medical School, Teviot Place,

Edinburgh EH8 9AG, UK

e-mail: jeremy.walker@ed.ac.uk

D. H. Brewster • C. M. Fischbacher • D. L. Stockton Information Services Division, NHS National Services Scotland, 1 South Gyle Crescent, Edinburgh, UK

H. M. Colhoun · G. P. Leese

Biomedical Research Institute, University of Dundee,

Ninewells Hospital \& Medical School, Dundee, UK

R. S. Lindsay $\cdot$ N. Sattar

British Heart Foundation Glasgow Cardiovascular Research Centre, University of Glasgow, 126 University Place,

Glasgow, UK

\section{J. A. McKnight}

Metabolic Unit, Western General Hospital, Crewe Road South, Edinburgh, UK

S. Philip

Grampian Diabetes Research Unit, Woolmanhill Hospital, Aberdeen, UK
Methods All people in Scotland aged 55-79 years diagnosed with any of the cancers of interest during the period 20012007 were identified and classified by the presence/absence of co-morbid type 2 diabetes. The influence of diabetes on cancer risk for each site was assessed via Poisson regression, initially with adjustment for age only, then adjusted for both age and socioeconomic status.

Results There were 4,285 incident cancers in people with type 2 diabetes. RR for any cancers (adjusted for age only) was $1.11(95 \%$ CI $1.05,1.17)$ for men and $1.33(1.28,1.40)$ for women. Corresponding values after additional adjustment for socioeconomic status were $1.10(1.04,1.15)$ and $1.31(1.25,1.38)$, respectively. RRs for individual cancer sites varied markedly.

Conclusions/interpretation Socioeconomic status was found to have little influence on the association between type 2 diabetes and cancer.

Keywords Cancer $\cdot$ Socioeconomic status
Abbreviations
NAFLD Non-alcoholic fatty liver disease
SCI-DC Scottish Care Information - Diabetes
Collaboration
SES Socioeconomic status
SIMD Scottish Index of Multiple Deprivation

\section{Introduction}

Diabetes has been linked with elevated risk of some cancers, including those of the pancreas, colon/rectum, liver, breast, endometrium and bladder [1]. Conversely, the incidence of prostate cancer is reduced in men with diabetes [1]. Postulated mechanisms for relationships between diabetes and cancer include shared risk factors (e.g. obesity) [2], metabolic 
abnormalities associated with diabetes [3], and the effects of diabetes treatments [3]. Diabetes is associated with lower socioeconomic status (SES) [4]; many cancers also exhibit social patterning. Consequently, relationships between diabetes and cancer may be confounded by SES. However, not all studies have adjusted for SES when assessing the risk of cancer associated with diabetes. Ogunleye and colleagues [5] investigated the association between diabetes and cancer using data from the Tayside region in Scotland and reported no evidence of confounding by SES. The present study investigated associations between diabetes and cancer using a similar approach but with three refinements, namely (1) exploiting data for the whole of Scotland (population approximately 5.1 million), thus providing a substantially larger sample size; (2) using more recent data; and (3) generating separate estimates by sex. The objective was to investigate whether adjustment for SES materially altered the estimated relationships between diabetes and common cancers. The study was restricted to type 2 diabetes because numbers of people with incident type 1 diabetes in the age range featured in the study (55-79 years) were too small to permit meaningful assessment of the relationship between diabetes and cancer at some sites.

\section{Methods}

Data sources Data for people diagnosed with diabetes in Scotland were obtained from the Scottish Care Information Diabetes Collaboration (SCI-DC) dataset [6]. This holds clinical and demographic data on almost all patients with diabetes in Scotland. The study was based on an extract of SCI-DC data performed in 2008; the latest complete calendar year covered was 2007. The investigation was restricted to people with incident type 2 diabetes who were aged 55-79 years for all or part of the period 2001 to 2007. This age range was selected to define a population with a high prevalence of type 2 diabetes and incidence of cancer, and to limit breast cancer to that occurring in post-menopausal women. Postcodes were used to assign a measure of SES, in the form of the Scottish Index of Multiple Deprivation (SIMD) [7]. For the purposes of this study, SIMD ranks for the 6,505 geographical areas were expressed as quintiles (the first and fifth quintiles representing the least and most deprived groups in the population, respectively).

The SCI-DC data were linked to national cancer registration records by the Information Services Division of National Services Scotland, who also provided cancer registration data covering the entire Scottish population, together with Scottish population numbers. Cancer registrations were analysed for 16 individual sites (five sex-specific e.g. breast, prostate; 11 common to both sexes) and for a composite category representing any cancer (ICD10 [www.who.int/classifications/icd/en/]; C00-97, excluding C44). Generation of the linked dataset was approved by the responsible regulatory bodies.

Statistical analysis Numbers of person-years accrued by people with incident type 2 diabetes in 2001-2007 were summed by age, sex and SIMD quintile. Follow-up started at the date of diabetes diagnosis, and people with a cancer registration that preceded diagnosis of diabetes were excluded. Follow-up ended at the date of cancer incidence, date of death (if within the study period) or at 31 December 2007. Person-years were included only for periods during which patients were within the specified age range (55-79 years). Numbers of incident cancers observed in people with diabetes were summed as for person-years. Where an individual experienced multiple tumours, only the chronologically earliest tumour was counted. Person-years at risk and incident cancer cases in the nondiabetic population were estimated as follows: first, personyears (calculated as above) for people with prevalent or incident diabetes within the study period were subtracted from the all-Scotland population totals for the period. Similarly, cancers diagnosed in people with diabetes were subtracted from the total number of people in Scotland with incident cancer at each site. The goal of this process was to permit estimation of incidence rates in a general population that was free from diabetes.

The RR of cancer associated with type 2 diabetes was estimated via Poisson regression models in which the number of people with cancer was predicted by age and diabetes status (type 2 diabetic vs non-diabetic population). Separate models were fitted for each sex and cancer site. All models were re-fitted to admit SES (SIMD quintile) as an additional predictor, the effect of introducing SES being formally assessed via likelihood ratio tests. All analysis was performed using SAS software (Version 9.2), SAS Institute, Cary, NC, USA.

\section{Results}

Results were based on data for 44,246 men and 36,592 women with incident type 2 diabetes who were aged 5579 years during the study period and did not have cancer at diabetes diagnosis. There were 2,445 incident cancers for all sites among men with type 2 diabetes, with over 400 incident prostate, lung and colorectal cancers. Among women with diabetes there were 1,840 incident cancers across all sites, with breast $(n=446)$, lung $(n=343)$ and colorectal $(n=$ $267)$ the most common cancers.

Estimates for the effect of type 2 diabetes on cancer incidence are shown in Table 1 (men) and Table 2 (women). Two sets of estimates are shown, representing 
Table 1 RR (with 95\% CI) of cancer at ages 55-79 in men (those with incident type 2 diabetes vs no diabetes); Scotland, 2001-2007

${ }^{a}$ Effect of SES in age- and SESadjusted model (likelihood ratio test, relative to model adjusted for age only)

\begin{tabular}{lllr}
\hline Cancer site (ICD10 code) & Age & Age+SES & $p$ value $^{\mathrm{a}}$ \\
\hline Prostate (C61) & $0.86(0.80,0.92)$ & $0.87(0.80,0.94)$ & $<0.001$ \\
Lung (C34) & $0.92(0.88,0.96)$ & $0.89(0.78,1.01)$ & $<0.001$ \\
Colorectal (C18-C20) & $1.24(1.12,1.39)$ & $1.24(1.12,1.37)$ & $<0.001$ \\
Head and neck (C00-C14, C30-C32) & $0.81(0.62,1.05)$ & $0.77(0.61,0.98)$ & $<0.001$ \\
Oesophagus (C15) & $1.00(0.79,1.27)$ & $0.98(0.77,1.25)$ & $<0.001$ \\
Bladder (C67) & $1.23(1.07,1.40)$ & $1.21(1.00,1.47)$ & $<0.001$ \\
Stomach (C16) & $1.06(0.85,1.32)$ & $1.03(0.83,1.28)$ & $<0.001$ \\
Non-Hodgkin's lymphoma (C82-C85) & $0.94(0.72,1.22)$ & $0.94(0.71,1.25)$ & 0.993 \\
Kidney (C64-C65) & $1.30(1.10,1.53)$ & $1.30(1.09,1.56)$ & 0.804 \\
Malignant melanoma (C43) & $1.03(0.77,1.37)$ & $1.05(0.75,1.46)$ & $<0.001$ \\
Pancreas (C25) & $2.89(2.60,3.22)$ & $2.85(2.39,3.41)$ & $<0.001$ \\
Liver (C22) & $3.65(2.82,4.72)$ & $3.54(2.83,4.43)$ & $<0.001$ \\
Any cancer (C00-C97 excl. C44) & $1.11(1.05,1.17)$ & $1.10(1.04,1.15)$ & 0.001 \\
\hline
\end{tabular}

RRs adjusted for age only, and RRs adjusted for age and SES. For a small number of cancers SES was not significantly predictive of incidence ( $p>0.05$; likelihood ratio test). Where SES was significant, risk estimates were in general not markedly affected by the introduction of SES to the model. In men, the highest RRs were observed for liver and pancreatic cancer but RRs adjusted for age and SES were also significantly elevated (at the conventional 5\% level) for colorectal, bladder and kidney cancer, and significantly reduced for prostate, head/neck and (marginally) lung cancer. Patterns of RR for women were broadly similar to those for men for the non-sex-specific cancers. There was also a significant excess risk of breast and endometrial cancer among women with diabetes compared with the non-diabetic population.

\section{Discussion}

We present estimates of the associations between type 2 diabetes and cancer in a national population-based retrospective cohort study. Our objective was to assess whether adjustment for the effect of SES materially impacted estimates of the RR of cancer associated with diabetes. As stated earlier, adjustment for SES has not universally been applied in previous studies in this area. We found that, although SES was in most cases significantly predictive of cancer incidence, RR estimates for the effect of diabetes generally showed only modest change after admission of SES to the models. It therefore appears that any differential risk of cancer that is associated with the presence of diabetes is not mainly attributable to confounding by SES in this population.
Table 2 RR (with 95\% CI) of cancer at ages 55-79 in women (those with incident type 2 diabetes vs no diabetes); Scotland, 2001-2007

${ }^{\mathrm{a}}$ Effect of SES in age- and SESadjusted model (likelihood ratio test, relative to model adjusted for age only)

\begin{tabular}{llrr}
\hline Cancer site (ICD10 code) & Age & Age+SES & $p$ value $^{\mathrm{a}}$ \\
\hline Breast (C50) & $1.20(1.04,1.38)$ & $1.21(1.08,1.36)$ & 0.003 \\
Lung (C34) & $1.12(1.02,1.23)$ & $1.05(0.93,1.19)$ & $<0.001$ \\
Colorectal (C18-C20) & $1.42(1.33,1.52)$ & $1.42(1.26,1.61)$ & 0.088 \\
Endometrium (C54-C55) & $1.93(1.46,2.53)$ & $1.94(1.59,2.37)$ & 0.178 \\
Ovary (C56) & $0.98(0.83,1.16)$ & $1.00(0.75,1.32)$ & 0.035 \\
Non-Hodgkin's lymphoma (C82-C85) & $1.17(0.82,1.67)$ & $1.18(0.90,1.55)$ & 0.162 \\
Malignant melanoma (C43) & $0.76(0.54,1.06)$ & $0.80(0.58,1.09)$ & $<0.001$ \\
Head and neck (C00-C14, C30-C32) & $0.95(0.68,1.34)$ & $0.90(0.60,1.34)$ & $<0.001$ \\
Pancreas (C25) & $3.70(2.98,4.60)$ & $3.64(3.03,4.38)$ & 0.022 \\
Oesophagus (C15) & $0.79(0.54,1.16)$ & $0.78(0.54,1.12)$ & $<0.001$ \\
Stomach (C16) & $1.35(1.06,1.72)$ & $1.30(0.97,1.75)$ & $<0.001$ \\
Kidney (C64-C65) & $1.84(1.48,2.28)$ & $1.81(1.34,2.44)$ & 0.198 \\
Bladder (C67) & $1.46(0.97,2.20)$ & $1.42(1.02,1.99)$ & $<0.001$ \\
Cervix uteri (C53) & $1.11(0.89,1.39)$ & $1.04(0.62,1.76)$ & $<0.001$ \\
Liver (C22) & $2.70(2.09,3.49)$ & $2.61(1.86,3.67)$ & 0.003 \\
Any cancer (C00-C97 excl. C44) & $1.33(1.28,1.40)$ & $1.31(1.25,1.38)$ & $<0.001$ \\
\hline
\end{tabular}


The Tayside study did not present sex-specific RR estimates but identified significantly raised risk of colon, pancreas and liver cancer among people with type 2 diabetes. These associations were also observed in this study, and accord with previous research [1]. The strong association between type 2 diabetes and liver cancer may reflect the consequences of non-alcoholic fatty liver disease (NAFLD), which can lead on to cirrhosis and hepatic cancer. Treatments such as metformin and pioglitazone that attenuate the effects of NAFLD may also impact on the outcome of liver cancer, although we were unable to test this hypothesis in this study. We also found significantly elevated risk for cancer of the kidney, bladder and endometrium that were not found in the Tayside study (perhaps reflecting smaller numbers of these cancers in that report). The present study also suggests reduced risk for prostate cancer, consistent with an earlier meta-analysis [8]. It therefore appears that, in extending the earlier study to harness national data, the findings accord more closely with the accumulated body of research in this field.

Strengths of this study include its use of complete data from a national, population-based register of people with diabetes. This provides sizeable patient populations, and avoids biases that can arise when conducting research in relatively small, selected samples e.g. hospital patients. A further strength is the availability of a wide-ranging measure of SES. Limitations include the inability to adjust for known confounders (such as diet and smoking), the 'multiple testing' issues arising from simultaneous evaluation of many individual estimates, and the lack of detailed data for the non-diabetic comparison group. One additional limitation is the possible influence of detection bias (the increased possibility of cancer being detected because the patient is under intensified clinical scrutiny following a recent diagnosis of diabetes, or vice versa) [9].

Possible mechanisms through which diabetes and cancer are inter-related include the influence of common risk factors, the effects of hyperglycaemia and hyperinsulinaemia and the potential influence of diabetes treatments on cancer risk. However, Suissa and Azoulay have demonstrated (using the example of metformin) that pharmacoepidemiological studies in the field of diabetes are commonly subject to time-related biases [10]. Consequently, the available evidence relating to the effects of diabetes treatments on cancer risk may be less reliable than is generally assumed. Irrespective of how these mechanisms contribute to the relationship between diabetes and cancer, our study suggests that their effects are largely independent of SES.

Acknowledgements This work is presented by the named authors on behalf of all members of the Scottish Diabetes Research Network Epidemiology Group. The data used are available for analysis thanks to the hard work of numerous NHS staff who enter the data, and people and organisations (the Scottish Care Information - Diabetes Collaboration [SCI-DC] Steering Group, the Scottish Diabetes Group, the Scottish Diabetes Survey Monitoring Group, the managed clinical network managers and staff in each Health Board) involved in setting up, maintaining and overseeing SCI-DC. Thanks are due also to staff of the Scottish Cancer Registry and to the Information Services Division (ISD) record linkage team.

Funding This work was funded by the European Foundation for the Study of Diabetes (grant number 90768) and the Wellcome Trust through the Scottish Health Informatics Programme (SHIP) Grant (grant number WT086113). Funding for diabetes register linkage was provided by the Scottish Government and the authors acknowledge the financial support of NHS Research Scotland (NRS), through the Scottish Diabetes Research Network.

Duality of interest The authors declare that there is no duality of interest associated with this manuscript.

Contribution statement JJW conducted the main data analysis and contributed to drafting of the manuscript. DHB, CMF and DLS contributed to interpretation of the data and critical revision of the text. GPL, RSL, NS and SP made contributions to the acquisition of data and revision of the manuscript. HMC and JAM contributed to the acquisition and interpretation of data, and to critical revision of the text. SHW designed the study, and contributed to acquisition and interpretation of data and drafting and revising the article. All authors approved the final version of the text.

\section{References}

1. Nicolucci A (2010) Epidemiological aspects of neoplasms in diabetes. Acta Diabetol 47:87-95

2. Giovannucci E, Harlan DM, Archer MC et al (2012) Diabetes and cancer: a consensus report. Diabetes Care 33:1674-1685

3. Simon D, Balkau B (2010) Diabetes mellitus, hyperglycaemia and cancer. Diabetes Metab 36:182-191

4. Brown AF, Ettner SL, Piette J et al (2004) Socioeconomic position and health among persons with diabetes mellitus: a conceptual framework and review of the literature. Epidemiol Rev 26:63-77

5. Ogunleye AA, Ogston SA, Morris AD, Evans JMM (2009) A cohort study of the risk of cancer associated with type 2 diabetes. Br J Cancer 101:1199-1201

6. McKnight JA, Morris AD, Clinet D, Peden N, Fischbacher C, Wild S (2008) Implementing a national quality assurance system for diabetes care: the Scottish Diabetes Survey 2001-2006. Diabet Med 25:743-746

7. Scottish Executive, Office of the Chief Statistician (2006) Scottish Index of Multiple Deprivation 2006: Technical Report. www.scotland.gov.uk/Resource/Doc/933/0041180.pdf. Accessed 18 April 2013

8. Kasper JS, Giovannucci E (2006) A meta-analysis of diabetes mellitus and the risk of prostate cancer. Cancer Epidemiol Biomarkers Prev 15:2056-2062

9. Johnson JA, Bowker SL, Richardson K, Marra CA (2011) Time-varying incidence of cancer after the onset of type 2 diabetes: evidence of potential detection bias. Diabetologia 54:2263-2271

10. Suissa S, Azoulay L (2012) Metformin and the risk of cancer. Diabetes Care 35:2665-2673 